\title{
Chapter 7 \\ Mainstreaming the Use of Life Cycle \\ Management in Small and Medium Sized \\ Enterprises Using a Sector Based and Regional Approach
}

\author{
Naeem Adibi, Vanessa Pasquet, Aubin Roy, Alice Salamon, Jodie Bricout, \\ Catherine Beutin, Quentin Renault, Marie Darul, François Xavier Callens, \\ Marc Haquette, Patrick Orlans, Jeanne Meillier, Joanne Boudehenn, \\ Sophie Reynaud, Sophie Cabaret, Christophe Bogaert, \\ and Christelle Demaretz
}

\begin{abstract}
Although Life Cycle Management (LCM) is becoming commonplace in larger corporations it is far from mainstream. To achieve sustainable production and consumption patterns, LCM needs to be taken up by whole supply chains that include small and medium sized enterprises (SMEs). From a business perspective, this represents a competitivity issue, as these SMEs are increasingly under pressure from clients and legislators to provide more information about the environmental impacts of their products, and to take responsibility for them both up and down the value chain. Therefore a sector based and regional approach is needed to foster the implementation of LCM in SMEs. This has been done in Northern France, where professional support organizations, including clusters, business federations and Chambers of Commerce, have come together under the auspices of the [avniR]
\end{abstract}

N. Adibi $(\bowtie) \bullet$ V. Pasquet $\bullet$ A. Roy $\bullet$ A. Salamon $\bullet$ J. Bricout

[avniR] Platform - cd2e, Rue de Bourgogne, Base du 11/19, 62750 Loos en Gohelle, France

e-mail:n.adibi@cd2e.com

C. Beutin

Aquimer competitivity cluster, 16 rue du Commandant Charcot,

62200 Boulogne-sur-Mer, France

Q. Renault

Agroe, excellence cluster, Quartier des Trois Parallèles, La Citadelle Avenue

du Mémorial des Fusillés, 62000 Arras, France

M. Darul $\bullet$ F.X. Callens $\bullet$ M. Haquette

cd2e excellence cluster, Rue de Bourgogne, Base du 11/19, 62750 Loos en Gohelle, France

P. Orlans

CETIM Technical Centre for the Mechanical Industry,

40 rue Eugène Jacquet, 59708 Marcq-en-Baroeul, France

(C) The Author(s) 2015

G. Sonnemann, M. Margni (eds.), Life Cycle Management,

LCA Compendium - The Complete World of Life Cycle Assessment,

DOI 10.1007/978-94-017-7221-1_7 
LCA Platform to explore ways to help businesses to adopt LCM. Nine pioneer sectors, textile, seafood, packaging, mechanical, food, wood, construction, recycling and renewable energies, have undertaken an ambitious project to integrate LCM into their business. The methodology for all nine sectors follows five major steps: benchmark, sector maturity assessment, needs identification, action plan and implementation.

Keywords Life cycle assessment $\bullet$ Life cycle management $\bullet$ Regional development - Sector-based approach $\bullet$ Small and medium sized enterprises $\bullet$ SMEs

\section{Introduction}

Life Cycle Management (LCM) has been defined by the SETAC working group as "an integrated framework of concepts, techniques and procedures to address environmental, economic, technological and social aspects of products and organizations to achieve continuous environmental improvement from a life cycle perspective" (Hunkeler et al. 2001). Supply chain actors have been pursuing to integrate LCM within their activities and collaborations for years.

Therefore, now, many major global companies have internal and external programs to assess and manage the sustainability performance of goods and services across the life cycle. LCM-related initiatives include sustainability parameters, like Key Performance Indicators (KPIs) and existing additional processes (NilssonLindén et al. 2014). Several private companies also finance collaborative life cycle research through initiatives such as SCORELCA, CIRAIG, Sustainability Consortium and UNEP/SETAC Life Cycle Initiative.

J. Meillier

UP-tex competitivity cluster, 40, rue Eugène Jacquet, 59708 Marcq-en-Baroeul, France

J. Boudehenn

Nord Picardie Bois, 34 B Rue Emile Zola, 59650 Villeneuve-d'Ascq, France

S. Reynaud

MATIKEM competitivity cluster, Parc scientifique de la Haute Borne,

9 rue Héloïse, 59650 Villeneuve d'Ascq, France

S. Cabaret

CCI Grand Lille, Place du Théâtre, 59000 Lille, France

C. Bogaert

ADEME Nord-Pas de Calais, Centre tertiaire de l'Arsenal,

20, rue du Prieuré, 59500 Douai, France

C. Demaretz

Nord-Pas de Calais Regional Council, 151 Avenue du Président Hoover, 59000 Lille, France 
LCM is also increasingly impacting public policy related to resources use and recycling, and more recently product-related environmental policies. For example, the European Commission launched "Building the Single Market for Green Products - Facilitating better information on the environmental performance of products and organizations" in April 2013. The Product/organizational Environmental Footprint (PEF/OEF) method (European Union 2013), published in April 2013, includes Commission recommendation on the use of common methods to measure and communicate the life cycle environmental performance of products and organizations. Within the related PEF/OEF pilot projects the Commission has engaged mainly big companies and industry associations.

Only few initiatives have been developed recently to help integrating LCM within SMEs in a sector. In 2013 the UNEP/SETAC Life Cycle Initiative supported eight pilot projects using Life Cycle Management Capability Maturity Model (LCM-CMM) in Cameroon, Uganda, South Africa, India, Brazil, Colombia, and Peru. As an example, results in Colombia showed that the companies could apply the LCM concepts to their organizations with limited technical support (Moreno et al. 2015).

Whilst the existing initiatives help to make significant progress towards sustainable production and consumption patterns, LCM needs to be taken up by whole supply chains that, by definition, include many small and medium sized enterprises (SMEs). From a business perspective, this represents a competitivity issue, as these SMEs are increasingly under pressure from clients and legislators to provide more information about the environmental impacts of their products, and to take responsibility for them both up and down the value chain. (Bricout et al. 2012)

\section{Context for the Study}

In this chapter we present a case study on how the use of Life Cycle Management can be mainstreamed in SMEs using a sector based and regional approach. A methodology for this purpose has been developed and applied in Northern France. It is a highly industrial region facing important challenges for sustainable development. The strong industrial past has affected the region through a complete reconversion from coal and heavy industry to other industrial and service sectors. Social challenges include the high population density (324 people per km² vs. 113 in France) with $95 \%$ living in urban communities. The environmental challenges this region is facing are diverse. The greenhouse gas emissions per capita are $30 \%$ higher compared to the French average. The share of renewable energy consumption is four times less than the national level. Only $1.9 \%$ renewable electricity (17.7\% France) is produced in the region and $16.4 \%$ of the surfaces area is artificial ( $8.8 \%$ France). Despite the loss of industrial activity over the past 50 years, Northern France remains the third largest industrial region in France with the fourth largest economic turnover. It is also a significant transport and logistics hub, with the densest road 


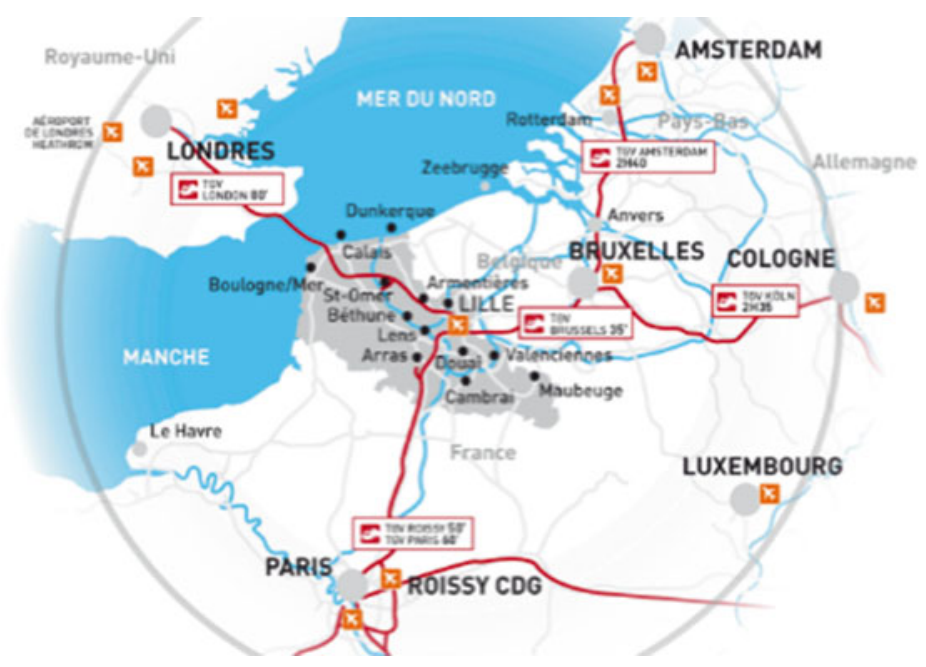

Fig. 7.1 The northern France region

network in Europe and significant rail and canal infrastructure (Nord-Pas de calais Regional Council 2013) (Fig. 7.1).

The Northern France region has been actively pursuing a transformation towards more sustainable economic models for many years. The non-for profit organization cd2e was established in 2002 to support this "eco-transition". As early as 2007, cd2e and their partners identified Life Cycle approaches as a needed decision-making tool to help local industry to implement Life Cycle Management. Cd2e created the [avniR] platform in 2009 to bring together multiple stakeholders around this challenging issue. This collective approach was needed to foster the supply and demand for LCA at the same time and has evolved from a focus on assessment to the support of companies in their efforts to improve their sustainability performance using LCM. On the LCM demand side, [avniR] works with industry clusters and public authorities to explain life cycle approaches and develop collaborative projects ([avnir] 2015). On the supply side, [avniR] provides training and a "hub" for academics and consultants to improve their capacity in Life Cycle Assessment, ecodesign and LCA based communication (Bjørn et al. 2013). Over 300 people have been trained in LCA/LCM through the platform, and more than 90 individual projects have been directly and indirectly supported.

Key milestones for the [avniR] platform include:

- 2011: first annual international [avniR] conference organized in Lille

- 2012: first ecodesign prize organized with different regional actors, in partnership with the Institut de Développement de Produits and Novae in Québec

- 2012: sectorial LCM studies launched in the textile, seafood, packaging and mechanical sectors 
- 2014: launch of a new governance based on different working groups to strengthen collaboration between the different stakeholders within the platform

The sectorial approaches launched in 2012 have the ambitious objective of moving beyond applying LCA studies in an isolated fashion, to mainstreaming life cycle management across entire sectors at the regional level, including the numerous SMEs present. The first "wave" of four sectors in 2012 (textile, seafood, packaging and mechanical) enabled the development of a common methodology to establish strategic action plans to mainstream LCM into business, education and research organizations. A second wave of sectors (food, wood, building and recycling/ end-of-life) undertook the process in 2013 and the renewable energies sector is developing their strategy in 2015.

This chapter presents the overall approach and the first results obtained in the mainstreaming of business Life Cycle Management practice and public Life Cycle Thinking awareness in a region through this, to our knowledge unique sectorial approach, whilst developing the necessary competence to transform awareness into action by stakeholders at different levels of influence.

\section{Approach}

Most SMEs lack the financial capacity or human resources to implement LCM on their own. Therefore, they need to work with support organizations at the regional level, and in general they are ready to work with other companies of their sector.

In Northern France, professional support organizations, including clusters, business federations and Chambers of Commerce, have come together under the auspices of the [avniR] LCA Platform to explore ways to help their businesses adopt LCM. The strategy for achieving this is based on the following key concepts (Adibi and Bricout 2012):

1. LCM capacity should be built into existing support organizations, so that businesses receive advice and tools from organizations that they already know and trust

2. Tools and actions to support LCM integration need to be adapted to different sectors to make them as relevant as possible to SMEs

3. Training and research capacity, also within universities and public research centers, needs to be developed to respond to needs of businesses

Before starting the process, there is an identification of the most strategic sectors to help integration of LCM within the region. The economic, environmental and social characteristics of the sector are considered jointly with the difficulty of mobilizing the sector (level of motivation/awareness and the existence of well identified actors for dissemination). 


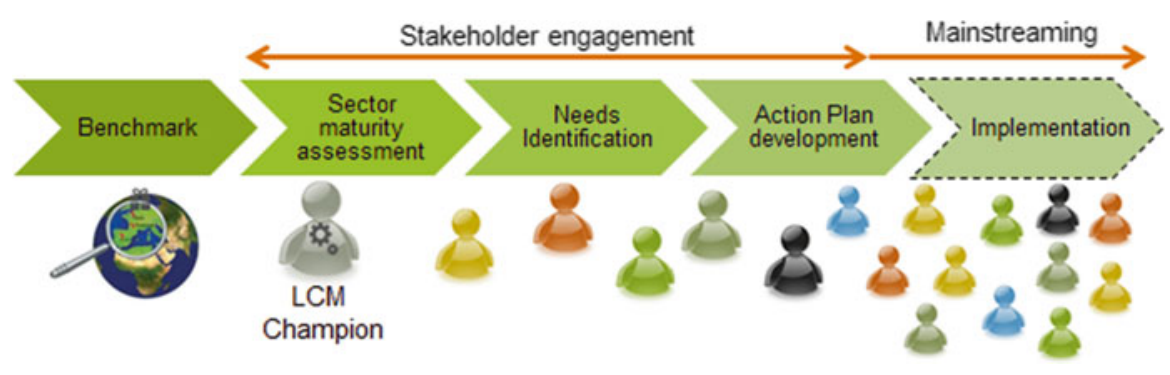

Fig. 7.2 Sector strategic action plan development methodology

For the sectors, existing support organizations are identified in a second step. We selected competitivity and excellence clusters, as they include innovation in their scope to help emerging new and innovative products and markets based on the implementation of LCM approaches. Once these organizations are identified, one reference person is designated for each sector. The different reference persons from the sectors, called "Life Cycle Champions", are creating an active network.

Champions are first trained on LCA and then on LCM approaches. A specific training of two days has been developed and delivered by the [avniR] platform. The training covers an advanced introduction to LCA, a detailed presentation of sectorial approaches and different steps and critical points to better integrate LCM within sectors based on the first sectorial experiences.

Based on [avniR] experience, it is strongly recommended to assure the presence of the same persons during the entire process through the implementation phase. When a change of person occurs, the step-by-step capitalization of the results becomes essential and the hierarchy within the structure (e.g., the president of the cluster) has to be extremely motivated to assure the continuation of the process.

All sectors follow the same general process for developing their LCM Action Plan, as shown in Fig. 7.2. The approach as presented in this chapter is elaborated within mid-long term vision and is not applicable within a short-term prospective.

Five major steps are followed within the process:

1. Benchmark

2. Sector maturity assessment

3. Needs identification

4. Action plan

5. Implementation

\subsection{Benchmark}

The benchmark process starts with the identification of the sector specific characteristics and a detailed mapping of the sector actors. For some of the sectors with very complex structure, the mapping phase is essential (e.g., building, recycling and packaging). The mapping helps to identify the major players including, businesses, 
education and research organizations as well as the interactions with institutions and authorities.

The sectors are very different from one another regarding the characteristics and the specificities that need to be identified during the benchmark phase. The major differences to be considered are detailed below.

\subsubsection{Structure of the Sector}

Some sectors include mostly very small and medium companies, whilst others may be dominated by big or medium enterprises or a mix of both. The type and size of organizations influence the way they will apply LCM. Also attributed resources are often related to the size of the structures and the turnover.

\subsubsection{Organization of the Sector}

Big companies that may not physically be present within the region sometimes drive sectors, for example, the mechanic sector has a very significant presence of purchasing/supplier relations. For some other sectors there might be a very logical and complementary chain of actors, varying from one country to another or from one region to another, as, for instance, for the building sector. In some sectors, companies (small, medium or big) may actually control the overall supply chain of their product.

\subsubsection{Product, Organization and Process Oriented Vision}

Another very import factor that may change completely the way sectors are responding to LCM is the way they look at the product. In some sectors, products are in the center of the business efforts, such as for the case of textile, fish and food. In others, processes are dominant, such as for the recycling sector. In most cases, for those sectors with a dominant process and organization vision, the role of actors and the relevance of products need to be clarified to help them uptake LCM.

Once a detailed mapping has been done, the next step within the benchmarking is to identify LCM initiatives, tools and case studies relevant to each sector, focusing on, but not limited to West Europe. This phase also includes a hotspot analysis based on existing LCAs within the sector. Figure 7.3 shows a list of major points considered within the benchmarking phase.

\subsection{Sector Maturity Assessment}

The maturity assessment aims at understanding the maturity of the actors of a sector in relation to LCM practices. In this way each sector and their respective actors can be trained corresponding to their needs in order to build LCM capabilities as quickly and efficiently as possible. 

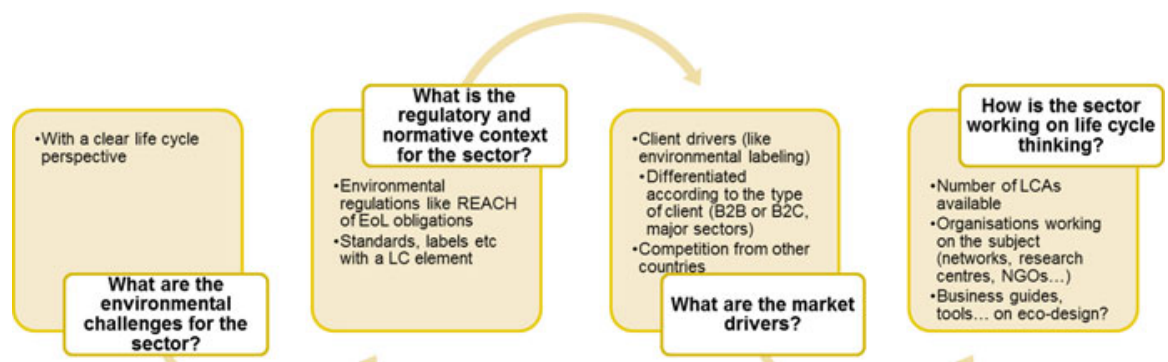

Fig. 7.3 Benchmarking

The first step for the maturity assessment is the sampling phase. Considering the mapping of actors (throughout the supply chains) and the structure of the sector (small, medium and big companies) a sample of the actors is selected. The sample covers different product/material groups within a given sector (e.g., food, meat, fruits, vegetables, drinks, etc. or the recycling of plastics, metals, wood etc.) to the extent possible within the region. In most cases, a sample covering $20-40 \%$ of the actors is sufficiently representative to formulate conclusions. Table 7.1 provides the key figures of sampling done for the textile, packaging, seafood and mechanical sectors.

Table 7.1 Key figures of sampling

\begin{tabular}{l|l|l|l|l}
\hline & Textile & Packaging & Seafood & Mechanical \\
\hline Companies concerned by the study & 200 & 90 & 130 & 750 \\
\hline Companies mobilized & $53(26 \%)$ & $32(36 \%)$ & $37(28 \%)$ & $201(27 \%)$ \\
\hline Surveyed & 17 & - & 17 & - \\
\hline Interviewed & 28 & 21 & 20 & 201 \\
\hline Active participation (workshops) & 8 & 11 & - & - \\
\hline $\begin{array}{l}\text { Researchers, universities, training } \\
\text { organizations, etc. }\end{array}$ & 7 & 4 & 16 & 8 \\
\hline Institution partners, federations, etc. & 10 & 14 & 8 & 15 \\
\hline Total & 70 & 50 & 61 & 224 \\
\hline
\end{tabular}

The maturity assessment of businesses, education bodies and research centers in the region in relation to LCM practices is undertaken via interviews with key stakeholders. To assess the maturity of the sectors, a baseline method has to be adapted for each sector and applied. We developed an adaptable baseline method called "[avniR] sectorial framework" (Adibi et al. 2012) based on the first wave of sectorial approaches and other methods developed for a similar purpose such as the Life Cycle Management Capability Maturity Model used by The UNEP/SETAC Life Cycle Initiative to promote LCM capability in different parts of the world.

The four methods used in the first wave of sectorial studies (textile, packaging, seafood and mechanical sectors) were developed independently and without applying 
any common framework. Based on the results of these studies and the feedback of responding companies and organizations, more sub-criteria were added. In the same time, the study of additional methods (CMM UNEP, etc.) provided a more exhaustive understanding of existing assessment methods. The [avniR] sectorial framework was finalized considering both experiences from the first wave pilot and other maturity assessment methods. The framework of the assessment method takes into account five main criteria:

1. Company global strategy for LCM

2. Challenges associated to LCA based approaches

3. Eco-design/LCA maturity approach

4. Resources dedicated to LCM

5. Continual improvement and communication efforts

The framework was applied within the second wave and was very successful to give a clear overview of the sectors' maturity. The method also helped through an indirect harmonization of the approach within different sectors.

The interest to develop the method was also to provide a common baseline that would allow later to monitor the advance of the sectors, once the implementation phase starts. In addition, the maturity assessment helped different sectors to better understand the action plans of other sectors, since not all of them started at the same maturity level, nor had the same need for training and capacity building support.

\subsection{Needs Identification}

The need identification considers two major aspects:

1. Benchmarking (national and international on the existing LCM capacities: LCM initiatives, tools and case studies relevant to each sector at global level and results of the hotspots analysis)

2. Sector maturity assessment results based on Sect. 3.2

For the needs identification, the LCM champion involves relevant stakeholders to build up a SWOT matrix to identify and categorize significant internal factors (i.e. strengths and weaknesses) and external factors (i.e. opportunities and threats) to integrate LCM in their sector. In order to better prepare and facilitate this phase, some preparatory documents are sent in advance to different stakeholders to facilitate their participation during the SWOT preparation.

Stakeholder engagement is a key aspect of the needs identification and a solid baseline for the action plan development that fits the needs of the sector. It is especially important for the implementation phase as the actions are "owned" by the participants. The step is also very important to educate the new stakeholders joining the process.

By the end of this phase the results of the first three phases are presented in an open meeting with all stakeholders. The aim is to make more and more stakeholders familiar with the process and findings, and motivate them to contribute to a successful implementation in the later stages. 


\subsection{Action Plan}

Each sector develops very detailed action plans based on the SWOT matrix and the identified needs and expectations. The experience with the first wave helped to develop major common indicators related to each action.

A non-exhaustive list of major common indicators is provided here: primary target, title description, goals, costs, time frame, potential action leader, starting time and duration, frequency, priority and management indicators.

In addition and to help cross cutting actions to be developed, actions are grouped in three major categories:

1. Sector specific actions

2. Replicable actions (to one or more sectors)

3. Cross cutting actions (joint actions of two or more sectors)

\subsection{Implementation}

The LCM action plans are developed in parallel, enabling the sectors to identify cross cutting actions. This process is managed by the regional LCA Platform [avniR], through the network of "Life Cycle Champions". Champions have been trained in the nine sectors; they meet regularly to exchange experiences in implementing life cycle approaches, identify cross cutting projects and to monitor sustainability performance advances within different sectors. The study was done through different waves. The latest sectors incorporated learnings from the first wave of sectors are shown in Fig. 7.4. Several actions are ongoing and some are finalized within different sectors. The results show significant improvements in SMEs within most sectors. The efficiency and effectiveness of the actions are monitored within a scoreboard developed together with LCM champions. Unfortunately, due to confidentiality restrictions, at the moment, no detailed results can be shown to highlight the improvements of the sustainability performance of products, processes and organizations achieved within the case study of Northern France.

\section{Conclusions and Outlook}

The chapter describes an innovative approach for mainstreaming LCM that is able to leverage sectorial and regional networks in order to help overcome barriers to the implementation of Life Cycle Management. From a business perspective, integration with existing professional organizations confirmed that SMEs access advice 

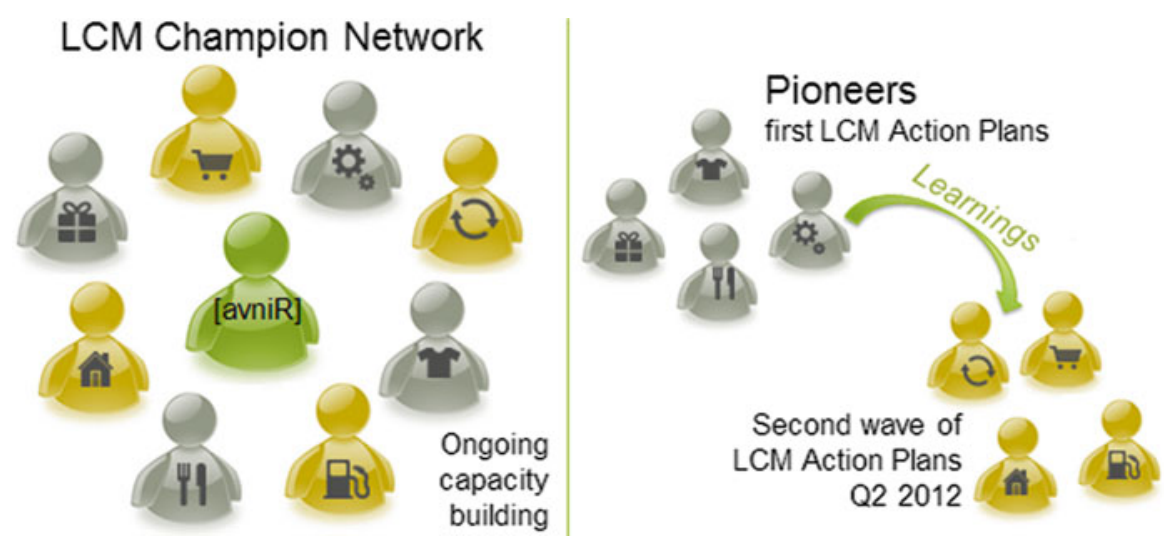

Fig. 7.4 The LCM champion network

and tools through organizations that they already know and trust. Working with several sectors in parallel through the Life Cycle Champion network encourages a multidisciplinary approach, essential to improving decision making across entire supply chains.

The results that can be reused elsewhere include new developments, such as a methodology to evaluate the maturity of sectors adaptable for different sectors and other regions in order to foster the implementation of Life Cycle Management worldwide.

In addition a very detailed action plan is developed for large scale roll-out of Life Cycle approaches in business and higher education. Each action plan aims to plan for the generalized roll-out of LCM in businesses across all industry sectors in the region. The engagement of stakeholders ensures that the benefits and effects will be perpetuated beyond the implementation phase.

In order to widely test and improve the methodology, a large scale project integrating regions from other European countries (Belgium, Portugal and Spain) is ongoing (Life Cycle in Practice - LIFE+ Funds). It aims to apply this approach in different regions, to improve the method and to validate the conclusions.

It is planned to publish the improvements achieved with regard to the sustainability performance of products, processes and organizations of at least one sector within the case study of Northern France, once confidentially agreements have been finalized.

Acknowledgements Authors would like to express sincere thanks to Christian Traisnel and Eric Kniaz for all their valuable feedbacks. The approach described is supported by the Nord-Pas de Calais Regional Council and the French environment agency, ADEME. 
Open Access This chapter is distributed under the terms of the Creative Commons Attribution Noncommercial License, which permits any noncommercial use, distribution, and reproduction in any medium, provided the original author(s) and source are credited.

\section{References}

[avniR] (2015) Etudes filières. http://www.avnir.org/FR/Etudes-filieres-227.html. Accessed 12 Mar 2015

Adibi N, Bricout J (2012) Mainstreaming life cycle approaches in northern France through the collaborative platform [avniR]. SETAC 18th LCA case study symposium, Copenhagen, p 164

Adibi $\mathrm{N}$ et al (2012) Introducing a sectorial framework to better evaluate life cycle thinking maturity. In: Proceedings of the 2nd international [avniR] annual conference, Lille, 2012

Bjørn A et al (2013) Mapping and characterization of LCA networks. Int J Life Cycle Assess 18(4):812-827

Bricout J et al (2012) Mainstreaming life cycle management: using a sector based and regional approach in northern France in the textile, seafood, packaging and mechanical sectors. In: Proceedings of the SETAC Europe annual meeting, Berlin

European Union (2013) PEF OEF methods. Off J Eur Union 56

Hunkeler D et al (2001) Life cycle management: bridging the gap between science and application. Int J Life Cycle Assess 6(6):384-390

Moreno OAV (2015) Implementation of life cycle management practices in a cluster of companies in Bogota, Colombia. Int J Life Cycle Assess. doi:10.1007/s11367-015-0875-x

Nilsson-Lindén H (2014) Organizing life cycle management in practice: challenges of a multinational manufacturing corporation. Int J Life Cycle Assess. doi:10.1007/s11367-014-0818-y

Nord-Pas de calais Regional Council (2013) La strategie regionale CLIMAT volet CLIMAT du SRADDT 